\title{
Relationship Between Metabolic Fitness and Performance in Police Occupational Tasks
}

\author{
Elisa F. D. Canetti ${ }^{1,2} \cdot$ J. Jay Dawes ${ }^{3} \cdot$ Phoebe H. Drysdale $^{1} \cdot$ Robert Lockie $^{4} \cdot$ Charlie Kornhauser $^{5} \cdot$ Ryan Holmes $^{5}$. \\ Ben Schram ${ }^{1,2} \cdot$ Robin M. Orr ${ }^{1,2}$
}

Received: 24 November 2019 / Accepted: 16 April 2020 / Published online: 20 May 2020

(C) The Author(s) 2020

\begin{abstract}
Purpose To determine the relationship between metabolic fitness test scores and performance in police occupational tasks. Methods Retrospective data from 106 law enforcement officers who completed two metabolic fitness assessments [anaerobic: a 20-m (10-m and 20-m splits) sprint, aerobic: $20 \mathrm{~m}$ Multi-Stage Fitness Test (20 m-MSFT)] and three routine occupational tasks [1.22 m Fence jump (FJ), 8.5 m Victim Drag (VD with $102.3 \mathrm{~kg}$ ) and "Get-up" (GU)] were collected. A Person's correlation coefficient and a standard multiple regression were used to assess the relationship between and predictive ability of, respectively, the fitness tests scores and performance on occupational tasks.

Results There was a significant positive correlation between the $10-\mathrm{m}$ sprint scores and FJ $(r=0.524, P<0.001)$, VD $(r=0.528, P<0.001)$ and GU $(r=0.486, P<0.001)$. Similarly, the $20-\mathrm{m}$ sprint scores were significantly and moderately correlated with FJ $(r=0.573, P<0.001)$, VD $(r=0.603, P<0.001)$ and GU $(r=0.533, P<0.001)$. These anaerobic tests' scores predicted approximately $40 \%$ of the performance in the occupational tasks analysed. The MSFT fitness assessment had a small, albeit significant negative correlation with the three tasks: $\mathrm{FJ}(r=-0.360, P<0.001)$, VD $(r=-0.266, P=0.012)$ and $\mathrm{GU}(r=-0.375, P<0.001)$.

Conclusion High levels of metabolic fitness correlated with faster performance in police officer occupational tasks, particularly ones of an anaerobic nature (i.e. the maximal sprint tests). In addition to typical aerobic fitness conditioning, anaerobic fitness conditioning could be vital for optimal performance in physically demanding tasks required of police officer.
\end{abstract}

Keywords Police $\cdot$ Occupational health $\cdot$ Physical fitness $\cdot$ Work performance $\cdot$ Rehabilitation

Elisa F. D. Canetti

ecanetti@bond.edu.au

1 Faculty of Health Sciences and Medicine, Bond Institute of Health and Sport, Bond University, 2 Promethean Way, Gold Coast, QLD 4226, Australia

2 Tactical Research Unit, Bond University, Gold Coast, QLD 4226, Australia

3 School of Kinesiology, Applied Health and Recreation, Oklahoma State University, 183 Colvin Recreation Center, Stillwater, OK 74078, USA

4 California State Fullerton, 800 N. State College Blvd, Fullerton, CA 92831-3599, USA

5 Colorado State Patrol, Lakewood, CO, USA

\section{Introduction}

Law enforcement officers provide service, security and protection for the population. The demands of police duties are varied [35]. Periods of sedentarism, where clerical and administrative tasks are performed (e.g. report writing) are interrupted by periods of high intensity efforts. These include patrolling large areas, climbing or bounding over obstacles, making arrests whilst carrying up to $10 \mathrm{~kg}$ in additional loads and reacting to unpredictable environmental strains $[2,28,33]$. The paradox is that such duties are not performed frequently enough to maintain appropriate levels of conditioning and preparedness for peak job performance. As such, police officers may lack the required occupational fitness to perform their duties safely and effectively [37].

Researchers have shown that fitness measures can be designed to replicate the demands of police duties in terms of aerobic capacity, anaerobic capacity, muscular strength 
and endurance [33]. Establishment of fitness standards within this population may increase an officer's ability to cope with the overall physical stress they may be faced with while performing essential occupational tasks [3, 31]. Moreover, such fitness may increase an officer's physical and mental preparedness for complicated tasks, ability to maintain alertness, recognise warning signs of impending violence, work in unpredictable environments, and maintain good interpersonal skills with members of the public $[26,28]$. This demonstrates a strong need for all police to develop and maintain high levels of fitness in all aspects of the job.

The potential for reduced fitness, combined with the known occupational health and safety risks faced daily by police officers [29], may increase an officer's risk of injury or illness. It has been discussed in previous reports that police officers have a higher risk of incurring a workrelated injury or illness than most other occupations [34]. Varvarigou et al. [47] reviewed 4553 on-duty police fatalities between 1984 and 2010 and identified 441 cases of sudden cardiac death. In this study, common duties of pursuit and restrains/altercations increased the relative risk of sudden cardiac death by 25.8 (95\% CI 18.4-36.2) and 40.6 (95\% CI 30.9-53.3), respectively, compared to routine/ non-emergency duties [47]. Metabolic, in particular, cardiorespiratory fitness (quantified by $\mathrm{VO}_{2 \max }$ ), is a known predictor of sudden cardiac death, where an increase of one metabolic equivalent in cardiorespiratory fitness may reduce the risk of sudden cardiac death by $22 \%$ [23].

Despite having identified that fitness is essential for performing specific policing tasks, to our knowledge, few researchers have reported strictly on the metabolic physical fitness levels of police officers specifically in relation to correlating performance in physical fitness tests and performance on routine job-related tasks [3, 34]. It is hypothesised that due to the explosive nature of many police duties, anaerobic capacity would have a larger correlation to occupational task performance when compared to aerobic capacity. Still, the ability to repeat the shortduration explosive duties also requires contribution of oxidative metabolism $[15,40]$, reported to contribute up to $40 \%$ of the energy required for the final repetitions [30]. Further, individuals with greater aerobic capacity (quantified by $\mathrm{VO}_{2 \max }$ ) have a superior ability to endure and resist fatigue during repeated short-duration exercises [4-6], such as sprints. A better understanding of the relationships between metabolic fitness and occupational tasks would enable police officers to ensure that they are adequately prepared for the physical aspects of the job and increase performance in routine operational duties. Thus, the aim of this study was to investigate whether a relationship exists between police officers' metabolic fitness and occupational task performance.

\section{Methods}

Retrospective data for male and female law enforcement officers from a US law enforcement agency (LEA) were provided for analysis. The data included specific metabolic field-based performance tests [(20 m Multi-Stage Fitness Test (20 m-MSFT) shuttle run, $10 \mathrm{~m}$ and $20 \mathrm{~m}$ sprints] and three routine occupational tasks (48" Fence jump, $8.5 \mathrm{~m}$ Victim Drag and "Get-up").

\section{Subjects}

US law enforcement officers $(N=106$, males $=101$, females $=5$ ) volunteered to participate in the study. Given that this was a sample of convenience, a post hoc power analysis was conducted using $G^{*}$ Power 3 [14] to test the correlations between the two measures using a two-tailed test, a medium effect size $(d=0.50)$, and an alpha of 0.05 . Result showed that a total sample of 106 participants achieved a power of 0.893 . All officers were employed by a US State Police department. Officers were recruited by the training staff by email invitation. Officers were selected using a random number generator (Google, LLC) in order to attain a cross-sectional cohort reflective of this population. Specific information related to the number of officers that declined to participate was not made available to the investigators. Officers diagnosed with any physical injury or illness that prevented them to perform the required tasks were excluded. Nutritional status, tobacco, supplement and medication usage, as well activity level on the days leading up to this test were not controlled. Officers were asked to maintain their normal routine in the days leading up to the assessment to minimize the risk of confounding variables. Prior to testing and analysis, approval for the use of the data was obtained from the Oklahoma State University Institutional Review Board (OSU IRB ED-19-146-STW) for human subjects, and the Bond University Human Research Ethics Committee (Bond HREC RO1927).

\section{Procedures}

The administration of fitness protocols and occupational tasks, and the collection of data were facilitated and captured by the staff of the LEA where the research took place. All assessing staff were trained by a Tactical Strength and Conditioning Facilitator who then verified the proficiency of the staff in the conduct of the assessments. Fitness assessments and occupational task simulations were conducted indoors at the LEA's training facility. Due to the high number of officers employed by the agency, multiple testing dates were scheduled throughout a three-month time 
frame (March-May). All the testing data in this study were collected in the calendar year 2016. All officers were fitted with a heart rate monitor (Polar Electro Inc., Lake Success, NY, USA) and completed a self-selected 5-10-min warmup prior to commencement of fitness assessments. Fitness assessments were conducted from least to most physically demanding to minimise the effect of fatigue on subsequent tests. Recovery periods were decided by the officers individually, ranging between 3 and $5 \mathrm{~min}$. These metabolic tests were part of an agency-required battery of test involving other physical fitness tests (i.e. strength tests). Officers were then asked to change into individual weighted tactical uniform, and further complete another set of simulated occupational tasks [fence jump (FJ), victim drag (VD) and get-up (GU)]. The tests included in the assessment are detailed below in the order in which they were performed. As all of the tests utilized were part of standard operating procedures for this agency, no familiarization trials were required. The following fitness tests were performed in the order presented.

\section{Timed $10 \mathrm{~m}$ and $20 \mathrm{~m}$ Sprints}

A $20 \mathrm{~m}$ course was set up inside the gymnasium. A laseractivated timing gate (TC-System, Brower Timing Systems, Draper, UT, USA) was placed at the start, $10 \mathrm{~m}$ and the end at $20 \mathrm{~m}$. Officers were instructed to position themselves at first timing gate and get set (without breaking the laser plane). On command officers were required to sprint as fast as they could to the end laser gate without slowing down or stopping. The time taken to complete the test was recorded by the timing system, as the officer ran through the first and last laser plane. The $10 \mathrm{~m}$ timing gate was set up half-way as to measure the $10 \mathrm{~m}$ split time.

\section{$22 \mathrm{~m}$ Fence Jump}

A free-standing steel chain link fence was constructed at a height of 1.22 by $2.21 \mathrm{~m}$ wide. The fence was rigidity positioned on hardwood flooring of the gymnasium for repeatability and safety. A laser-activated timing gate (TCSystem, Brower Timing Systems, Draper, UT, USA) was placed exactly 5-m away from the fence on either side to mark a start and end points. Officers were instructed to position themselves at the first timing gate and get set (without breaking the laser plane). On command, officers were required to run up to the fence, get over the fence as quickly and safely as possible, and then run through the end timing gate without slowing down or stopping prior to breaking the laser plane. The time taken to complete the exercise was recorded by the laser system, as the officer ran through the first and last laser plane. Officers were allowed two trials, with the best time from these trials used for analysis.

\section{0 m Multi-Stage Fitness Test (20 m-MSFT)}

The $20 \mathrm{~m}$ Multi-Stage Fitness Test (20 m-MSFT) is a standard multistage shuttle run test performed by each subject inside the gymnasium and was used to provide a prediction of the subject's aerobic power $\left(\mathrm{VO}_{2 \max }\right)$ [39]. This test is strongly linked to direct measures of aerobic capacity and is a commonly used field-based assessment, it was performed based on the criteria outlined by Léger and Lambert [24]. Officers were required to run back and forth between two lines marked on the ground spaced exactly $20 \mathrm{~m}$ apart. The speed of running for this test is standardized by pre-recorded auditory cues (beeps). The initial speed for the test is set at $8.5 \mathrm{~km} / \mathrm{h}$ and increases by $0.5 \mathrm{~km} / \mathrm{h}$ with each additional stage. All officers were required to complete the $20 \mathrm{~m}$ distance before every audible "beep" occurred, turn $180^{\circ}$ and again complete the $20 \mathrm{~m}$ in the opposite direction before the next audible "beep". This test was scored according to the final stage and shuttle (e.g. Stage 6.5) the participant was able to achieve. The test was terminated when the officer was unable to reach the next line twice in a row in accordance with the auditory "beep". Final scores by stage and shuttle were converted for total number of shuttles completed.

\section{5 m Victim (Dummy) Drag}

A long track of $8.5 \mathrm{~m}$ by $1.5 \mathrm{~m}$ was constructed on a level carpeted surface in LEA's gymnasium. A rescue-training dummy weighing $102 \mathrm{~kg}$ was placed at the starting line (marked by two plastic cones and laser-activated timing gates (TC-System, Brower Timing Systems, Draper, UT, USA). Officers were commanded to grasp the dummy by the arm and drag it the $8.5 \mathrm{~m}$ track as quickly as possible to the final timing gate. Officers started by holding the dummies hand and were instructed not to break the timing gates. Time taken to complete the task was the indicator of overall success of the officer's victim drag for this study.

\section{Get Up}

A "get up" was used to access how quickly an officer could respond to a certain stimulus from a supine position into a fighting stance, adopting a position ready for what may be about the face them. All subjects performed the "get up" as they had been trained with one assessor timing them. This exercise was assessed indoors on the defence training room mats. The exercise began with the subject lying supine 
with arms and legs apart. On command, the subject would, jump up from their floor position and resume a final fighting stance as quickly and safely as able. The assessor recorded how quickly the subject performed the exercise with a handheld stopwatch (Accusplit, Pleasanton, CA, USA), from the moment the officer moved to when they were steadily on two feet. Technique used to get up (e.g., blocking/shielding stance), technique safety and total time to finish were all recorded by the assessor. Total time was the measure analysed in this study.

\section{Statistical Analyses}

Statistical analyses were performed in SPSS ${ }^{\circledR}$ software (Version 26, IBM Corporation). Descriptive statistics were generated to identify physical characteristics of the subjects. Preliminary exploration of data revealed violation of normality. Data transformations were performed [45], and extreme outliers removed. A bivariate Persons' productmoment correlation coefficient $(r)$ was used to assess the relationship between the metabolic field-based performance tests and occupational tasks. Alpha levels were set at 0.05 a priori. The magnitude of the correlation coefficients were considered trivial $(r<0.1)$, small $(0.1<r<0.3)$, moderate $(0.3<r<0.5)$, large $(0.5<r<0.7)$, very large $(0.7<r<0.9)$, nearly perfect $(r>0.9)$ and perfect $(r=1.0)[19,20]$. A standard multiple regression was performed to assess if performance in occupation task could be predicted by fitness test scores. The adjusted $R^{2}$ value of each model was used to report the proportion of variance in the occupational task performance that were accounted for by the all the three fitness tests combined [1].

\section{Results}

One-hundred and six subjects were initially enrolled in the study. While there was no loss of subjects during the events, one male subject was excluded from the descriptive analysis due to incomplete data. Demographic and physical
Table 2 Performance on fitness and occupational tests

\begin{tabular}{lccc}
\hline Task & Mean & \multicolumn{2}{l}{$95 \% \mathrm{CI}$} \\
\cline { 3 - 4 } & & Lower & Upper \\
\hline MSFT (n of shuttles) & 39.6 & 35.8 & 43.3 \\
VO $_{2 \text { max }}$ (L/min) & 31.8 & 31.0 & 33.6 \\
10 m Sprint (s) & 2.3 & 2.2 & 2.3 \\
20 m Sprint (s) & 3.9 & 3.8 & 3.9 \\
Fence jump (s) & 5.6 & 5.3 & 5.9 \\
Victim drag (s) & 7.2 & 6.8 & 7.6 \\
Get up (s) & 2.7 & 2.6 & 2.9 \\
\hline
\end{tabular}

${ }^{\text {a }}$ Estimated $\mathrm{VO}_{2 \max }$ from MSFT [15]

characteristics of the remaining 105 subjects are displayed in Table 1.

The overall performance on the fitness tests and occupational tasks are reported in Table 2. The relationship between scores in the fitness tests and performance in the selected occupational tasks are depicted in Table 3. Missing or incomplete datasets were excluded from the correlation analysis.

Performance on both anaerobic fitness tests, $10 \mathrm{~m}$ and $20 \mathrm{~m}$ sprint, had a large positive statistically significant correlation to all occupational tasks (Table 3). Positive correlations indicated that faster time on these fitness variables were associated with faster times on occupational tasks, thus increased police officer performance.

The $20 \mathrm{~m}$-MSFT results yielded a small to moderate negative statistically significant correlation between all occupational tasks. Higher levels reached on the MSFT were correlated with faster times to complete the occupational tasks. Results from the standard multiple regression identified that, in combination, the fitness tests predicted $30 \%$ of the performance in the FJ $\left(R^{2}=0.331\right.$, adjusted $\left.R^{2}=0.306, F(3,83)=13.66, P<0.001\right)$. Similarly, $32 \%$ of the performance in the VD was predicted by the fitness tests $\left(R^{2}=0.347\right.$, adjusted $R^{2}=0.324, F(3,83)=14.71$, $P<0.001)$. Interestingly, $5 \%$ of the performance in VD was uniquely attributed to performance in the $20 \mathrm{~m}$ sprint

Table 1 Descriptive characteristics of officers

\begin{tabular}{|c|c|c|c|c|c|c|c|c|}
\hline & \multicolumn{4}{|c|}{ Male $(n=100)$} & \multicolumn{4}{|c|}{ Female $(n=5)$} \\
\hline & Mean & SD & $95 \%$ CI lower & $95 \%$ CI upper & Mean & SD & $95 \%$ CI lower & $95 \%$ CI upper \\
\hline Age (years) & 42.1 & 7.0 & 40.7 & 43.5 & 42.0 & 7.8 & 32.3 & 51.7 \\
\hline Height $(\mathrm{cm})$ & 179.1 & 7.4 & 177.6 & 180.5 & 168.7 & 6.9 & 160.1 & 177.2 \\
\hline Mass (kg) & 96.9 & 17.8 & 93.4 & 100.5 & 77.9 & 16.2 & 57.8 & 98.0 \\
\hline BMI $\left(\mathrm{kg} / \mathrm{m}^{2}\right)$ & 30.0 & 4.8 & 29.0 & 30.9 & 27.0 & 4.4 & 21.6 & 32.5 \\
\hline Duty weight (kg) & 106.4 & 17.7 & 102.9 & 110.0 & 89.2 & 18.0 & 67.0 & 111.4 \\
\hline Weight difference $(\mathrm{kg})$ & 9.5 & 1.8 & 9.1 & 9.8 & 11.3 & 2.5 & 8.2 & 14.4 \\
\hline
\end{tabular}


Table 3 Correlation between fitness tests and performance in occupational tasks

\begin{tabular}{llll}
\hline & 10 m Fence jump & Victim drag & Get-up \\
\hline 10 m Sprint & & & \\
Correlation coefficient $(r)$ & 0.524 & 0.528 & 0.486 \\
$95 \%$ Confidence intervals & $0.359,0.658$ & $0.363,0.661$ & $0.312,0.628$ \\
Sig. (2-tailed) & $<0.001$ & $<0.001$ & $<0.001$ \\
$n$ & 93 & 93 & 92 \\
20 m Sprint & & & \\
Correlation coefficient $(r)$ & 0.573 & 0.603 & 0.533 \\
$95 \%$ Confidence intervals & $0.420,0.694$ & $0.457,0.717$ & $0.370,0.664$ \\
Sig. (2-tailed) & $<0.001$ & $<0.001$ & $<0.001$ \\
$n$ & 95 & 95 & 94 \\
MSFT & & & -0.375 \\
Correlation coefficient $(r)$ & -0.360 & -0.266 & $-0.541,-0.181$ \\
$95 \%$ Confidence intervals & $-0.528,-0.165$ & $-0.449,-0.061$ & $<0.001$ \\
Sig. (2-tailed) & $<0.001$ & 0.012 & 89 \\
$n$ & 90 & 89 & \\
\hline
\end{tabular}

$r$ Pearson's correlation coefficient, $n$ number, MSFT multi-stage fitness test test $(P=0.013)$. The fitness tests predicted $26 \%$ of the performance in the $\mathrm{GU}\left(R^{2}=0.288\right.$, adjusted $R^{2}=0.262, F(3$, $82)=11.067, P<0.001)$.

\section{Discussion}

The results of this study suggest that high levels of metabolic fitness are correlated with better performance in police officer occupational tasks, particularly ones of anaerobic nature (i.e. the maximal sprint tests). Further, the tasks reported herein are designed to replicate occupational demands of policing, thus providing a pragmatic translation to police occupational performance.

Anaerobic fitness, assessed by the $10 \mathrm{~m}$ and $20 \mathrm{~m}$ sprints, was strongly associated with the performance of several police tasks. Completion of such short distances at maximal speed rely mostly on lower-limb power. This anaerobic characteristic of power is often assessed through a vertical jump (VJ) test. Orr et al. [36], measured the relationship between power, strength, and linear running speed amongst special weapons and tactics (SWAT) police officers. The authors reported a significant moderate relationship ( $r$ range $=-0.572$ to $-0.608, P<0.001$ ) between speed during the $5 \mathrm{~m}, 10 \mathrm{~m}$ and $20 \mathrm{~m}$ sprints to peak power measured via VJ. Recently, Moreno et al. [32] suggested that while VJ did contribute to a better performance in the VD $(r=0.209, P<0.05)$, the standing broad jump was more task specific $(r=0.609, P<0.001)$ as it produces horizontal power. Findings from this study support the contribution of power, both vertical and horizontal, to performance in the police tasks of FJ and VD, respectively. Thus, it can be concluded that the higher level of anaerobic power, assessed either through VJ or short duration sprints can predict performance in a number of police officer occupational tasks, such as rapidly sprinting to an injured victim, chasing a perpetrator over a short distance, or taking cover when under threat.

Results of this study demonstrate a small, yet significant, relationship between aerobic fitness, assessed through the $20 \mathrm{~m}$-MSFT, and performance in the occupational tasks assessed. The $20 \mathrm{~m}$-MSFT is an indicator of aerobic fitness $[17,42]$. The low correlation between the tasks and the $20 \mathrm{~m}$-MSFT observed herein, may be due to the explosive nature and short duration of the occupational tasks performed. Dawes et al. [10] investigated the relationship between physical fitness tests of patrol officers and a simulated occupational Physical Agility Test (PAT). The PAT consisted of several consecutive tasks with no rest periods, thus relying heavily on aerobic endurance. The researchers concluded that overall, the $20 \mathrm{~m}$-MSFT was the best predictor of performance in the PAT [10]. Such findings indicate that whilst aerobic capacity may not be highly correlated with single tasks, it is a crucial component of police officer fitness as (1) it is unlikely that in the line of duty only one short-burst task will be required of a police officer and (2) it is essential for recovery between short bouts of explosive power [43]. This supposition is supported by Decker et al. [12] who found that some police duties, such as attending a domestic violence situation, could last over 120 min with heart rate averaging $50 \%$ of maximum and reaching over $100 \%$ of maximum during this time. Moreover, aerobic endurance, intimately related to cardiovascular fitness [22, 25, 48], ensures overall health thus decreasing an officer's risk of injury or illness [41]. This health benefit is of note given that police officers are at a higher risk of 
cardiovascular disease when compared to the general public [18].

Metabolic fitness is vital for performance in police occupational tasks. Entry to the police force is often dependent on physical assessments of aerobic and anaerobic nature. Such selection is made for a variety of reasons and include as an early measure of police task potential [27] to determining injury risk during training [11]. Interestingly, whilst studies recommend a minimum aerobic capacity $\left(\mathrm{VO}_{2 \max }\right)$ of $45 \mathrm{~mL} / \mathrm{kg}$ for firefighters [16, 44], such recommendation is yet to be made for law enforcement officers [38].

Findings from this and other studies suggest that fitness measures such as aerobic capacity, muscular endurance and anaerobic power should be incorporated as an on-going requirement for police officers to ensure that optimal performance in occupational tasks is achieved [3, 9, 10, 31, 43]. The importance of this progression of fitness assessments beyond initial training is found in the study by Orr et al. [37] who found that, when aged matched against trainees, incumbent officers were less physically fit. The authors of this study suggest that this lower fitness of incumbent officers is partly due to the occupational requirements of officers which include barriers like shift work and long work hours [37].

This study is not without limitations. The fitness assessments were performed in self-selected training clothes whilst simulated occupational tasks were performed wearing full duty uniforms. Not only did this increase the average weight (Table 1), but the extra volume of a tactical vest could have impacted the performance in the occupational tasks. Researchers have identified significant biomechanical [46], metabolic [13] and overall performance decrements [13, 46] in law enforcement personnel when wearing body armour. Therefore, future research assessing fitness and occupational tasks should advocate for the use of body armour in both conditions, to ensure a realistic assessment of performance. Further, female representation in this study was low (4.7\%), hardly a true representation of females in the force, reported to be $12.3 \%$ of full-time sworn officers in US local police departments [21], $13.6 \%$ of sheriffs' offices [8] and $13.7 \%$ of federal law enforcement officers [7].

In conclusion, the results of the current study highlight that increased metabolic fitness correlated to better performance in police officer occupational tasks. The $10 \mathrm{~m}$ and $20 \mathrm{~m}$ sprints demonstrated a higher correlation to occupational tasks performed when compared to the $20 \mathrm{~m}$-MSFT. However, it should be noted that occupational tasks assessed in this study mainly relied upon anaerobic power thus, these measures should not be deemed superior indicators of occupational performance when compared to the $20 \mathrm{~m}$-MSFT but rather relevant and required for police officer task performance. Future studies aiming to use fitness measures to predict occupational task performance should incorporate a greater variety of occupational tasks that involve greater aerobic capacity, such as a timed long-distance run. The high physical demand of police tasks highlights the necessity of continuous metabolic fitness training and assessment to ensure optimal performance in policing duties and overall health. Findings of the current study suggest that these assessments may be of use during the recruitment and training of new police officers, may serve as a means of measuring fitness levels of existing officers, and may be of use to determine return-to-work capacity in police officers undergoing rehabilitation.

\section{Compliance with Ethical Standards}

Conflict of interest Authors EFDC, JJD, PHD, RL, CK, RH, BS and RO declare that they have no conflict of interest. This article's title and abstract are present on the Australian Physiotherapy Association-Transform Conference website and can be accessed on https:// transform.physio/wp-content/uploads/2019/09/Abstract_Book_Adela ide_2019.pdf.

Ethical approval All procedures followed were in accordance with the ethical standards of the responsible committee on human experimentation (institutional and national) and with the Helsinki Declaration of 1975 , as revised in 2000 .

Informed consent Informed consent was obtained from all participants included in the study.

Open Access This article is licensed under a Creative Commons Attribution 4.0 International License, which permits use, sharing, adaptation, distribution and reproduction in any medium or format, as long as you give appropriate credit to the original author(s) and the source, provide a link to the Creative Commons licence, and indicate if changes were made. The images or other third party material in this article are included in the article's Creative Commons licence, unless indicated otherwise in a credit line to the material. If material is not included in the article's Creative Commons licence and your intended use is not permitted by statutory regulation or exceeds the permitted use, you will need to obtain permission directly from the copyright holder. To view a copy of this licence, visit http://creativecommons.org/licenses/by/4.0/.

\section{References}

1. Allen P, Bennett K, Heritage B. SPSS statistics: a practical guide. 4th ed. Melbourne: Cengage; 2019. pp. 179-97

2. Beck B, Carstairs GL, Caldwell Odgers JN, Doyle TLA, Middleton KJ. Jerry can carriage is an effective predictor of stretcher carry performance. Ergonomics. 2015;59(6):813-20.

3. Beck AQ, Clasey JL, Yates JW, Koebke NC, Palmer TG, Abel MG. Relationship of physical fitness measures vs. occupational physical ability in campus law enforcement officers. J Strength Cond Res. 2015;29(8):2340.

4. Bishop D, Edge J. Determinants of repeated-sprint ability in females matched for single-sprint performance. Eur J Appl Physiol. 2006;97(4):373-9.

5. Bishop D, Edge J, Goodman C. Muscle buffer capacity and aerobic fitness are associated with repeated-sprint ability in women. Eur J Appl Physiol. 2004;92(4-5):540-7.

6. Bishop D, Girard O, Mendez-Villanueva A. Repeated-sprint ability—part II. Sports Med. 2011;41(9):741-56. 
7. Brooks C. Federal Law Enforcement Officers, 2016-Statistical Tables 2019. http://www.bjs.gov/index.cfm?ty=pbdet ail\&iid=6708. Accessed 11 Apr 2019.

8. Brooks C. Sheriffs' Offices, 2016: Personnel 2019. http://www.bjs. gov/index.cfm?ty=pbdetail\&iid=6707. Accessed 11 Apr 2019.

9. Dawes JJ. Description of the anthropometric and physiological profile of tactical officers. Oklahoma: Oklahoma State University; 2011.

10. Dawes JJ, Lindsay K, Bero J, Elder C, Kornhauser C, Holmes R. Physical fitness characteristics of high vs. low performers on an occupationally specific physical agility test for patrol officers. J Strength Cond Res. 2017;31(10):2808-15.

11. Dawes JJ, Lockie R, Orr RM, Pope R, Kornhauser C, Holmes R. Initial fitness testing scores as a predictor of police academy graduation. J Aust Strength Cond. 2019;27(4):30-7.

12. Decker A, Orr R, Pope R, Hinton B. Physiological demands of law enforcement occupational tasks in Australian police officers. J Aust Strength Cond. 2016;24(6):78-9.

13. DiVencenzo HR, Morgan AL, Laurent CM, Keylock KT. Metabolic demands of law enforcement personal protective equipment during exercise tasks. Ergonomics. 2014;57(11):1760-5.

14. Faul F, Erdfelder E, Lang A-G, Buchner A. G*Power 3: a flexible statistical power analysis program for the social, behavioral, and biomedical sciences. Behav Res Methods. 2007;39(2):175-91.

15. Girard O, Mendez-Villanueva A, Bishop D. Repeated-sprint ability-part I. Sports Med. 2011;41(8):673-94.

16. Gledhill N, Jamnik VK. Characterization of the physical demands of firefighting. Can J Sport Sci. 1992;17(3):207-13.

17. Hauschild VD, DeGroot DW, Hall SM, Grier TL, Deaver KD, Hauret KG, Jones BH. Fitness tests and occupational tasks of military interest: a systematic review of correlations. Occup Environ Med. 2017;74(2):144-53.

18. Hinton B, Stierli M, Orr R. Physiological issues related to law enforcement personnel. In: Alvar B, Sell K, Deuster P, editors. NSCA's essentials of tactical strength and conditioning. Champaign: Human Kinetics; 2017. p. 577-604.

19. Hopkins WG. Measures of reliability in sports medicine and science. Sports Med. 2000;30(1):1-15.

20. Hopkins WG, Marshall SW, Batterham AM, Hanin J. Progressive statistics for studies in sports medicine and exercise science. Med Sci Sports Exerc. 2009;41(1):3-13.

21. Hyland S, Davis E. Local Police Departments, 2016: Personnel 2019. http://www.bjs.gov/index.cfm?ty $=$ pbdetail $\&$ iid $=6706$. Accessed 11 Apr 2019.

22. Jones AM, Carter H. The effect of endurance training on parameters of aerobic fitness. Sports Med. 2000;29(6):373-86.

23. Laukkanen JA, Mäkikallio TH, Rauramaa R, Kiviniemi V, Ronkainen K, Kurl S. Cardiorespiratory fitness is related to the risk of sudden cardiac death: a population-based follow-up study. J Am Coll Cardiol. 2010;56(18):1476-83.

24. Leger LA, Lambert J. A maximal multistage 20-m shuttle run test to predictldot $\mathrm{VO}_{2 \max }$. Eur J Appl Physiol Occup Physiol. 1982;49(1):1-12.

25. Lin X, Zhang X, Guo J, Roberts CK, McKenzie S, Wu WC, Liu $\mathrm{S}$, Song Y. Effects of exercise training on cardiorespiratory fitness and biomarkers of cardiometabolic health: a systematic review and meta-analysis of randomized controlled trials. J Am Heart Assoc. 2015;4(7):e002014.

26. Lindberg AS, Oksa J, Gavhed D, Malm C. Field tests for evaluating the aerobic work capacity of firefighters. PLoS One. 2013;8(7):e68047.

27. Lockie RG, Dawes JJ, Balfany K, Gonzales CE, Beitzel MM, Dulla JM, Orr RM. Physical fitness characteristics that relate to work sample test battery performance in law enforcement recruits. Int J Environ Res Public Health. 2018;15(11).
28. Mayhew C. Occupational health and safety risks faced by police officers. Trends Issues Crime Crim Justice. 2001;196:1-6.

29. Mayhew C. Occupational health and safety risks faced by police officers. Trends \& Issues in Crime and Criminal Justice, vol. 169. Canberra: Australian Institute of Criminology; 2001. p. 1-6.

30. McGawley K. Anaerobic and aerobic contribution to two, $5 \times 6-\mathrm{s}$ repeated-sprint bouts. Coach Sport Sci J. 2008;3(2):52.

31. Michaelides MA, Parpa KM, Henry LJ, Thompson GB, Brown BS. Assessment of physical fitness aspects and their relationship to firefighters' job abilities. J Strength Cond Res. 2011;25(4):956-65.

32. Moreno M, Dulla J, Dawes JJ, Orr R, Cesario K, Lockie R. Lowerbody power and its relationship with body drag velocity in law enforcement recruits. Int J Exerc Sci. 2019;12(4):847-58.

33. Orr RM. The history of the soldier's load. Aust Army J. 2010;7(2):67-88.

34. Orr RM, Kukić F, Čvorović A, Koropanovski N, Janković R, Dawes JJ, Lockie RG. Associations between fitness measures and change of direction speeds with and without occupational loads in female police officers. Int J Environ Res Public Health. 2019;16(11).

35. Orr RM, Wilson A, Pope R, Hinton B. Profiling the routine tasks of police officers. J Aust Strength Cond. 2016;24(6):80.

36. Orr RM, Dawes JJ, Elder C, Krall K, Stierli M, Schilling B. Relationship between selected measures of power and strength and linear running speed amongst Special Weapons and Tactics police officers. J Aust Strength Cond. 2015;23(3):23.

37. Orr RM, Dawes JJ, Pope R, Terry J. Assessing differences in anthropometric and fitness characteristics between police academy cadets and incumbent officers. J Strength Cond Res. 2018;32(9):2632-41.

38. Pryor RR, Colburn D, Crill MT, Hostler DP, Suyama J. Fitness characteristics of a suburban special weapons and tactics team. J Strength Cond Res. 2012;26(3):252-7.

39. Ramsbottom R, Brewer J, Williams C. A progressive shuttle run test to estimate maximal oxygen uptake. Br J Sports Med. 1988;22(4): 141 .

40. Sanders GJ, Turner Z, Boos B, Peacock CA, Peveler W, Lipping A. Aerobic capacity is related to repeated sprint ability with sprint distances less than 40 meters. Int J Exerc Sci. 2017;10(2):197-204.

41. Schilling R, Colledge F, Ludyga S, Pühse U, Brand S, Gerber M. Does cardiorespiratory fitness moderate the association between occupational stress, cardiovascular risk, and mental health in police officers? Int J Environ Res Public Health. 2019;16(13).

42. Siddall A, Stevenson R, Turner P, Stokes K, Bilzon J. Development of role-related minimum cardiorespiratory fitness standards for firefighters and commanders. Ergonomics. 2016;59(10):1335-43.

43. Silk A, Savage R, Larsen B, Aisbett B. Identifying and characterising the physical demands for an Australian specialist policing unit. Appl Ergon. 2018;68:197-203.

44. Sothmann MS, Saupe K, Jasenof D, Blaney J. Heart rate response of firefighters to actual emergencies. Implications for cardiorespiratory fitness. J Occup Med. 1992;34(8):797-800.

45. Tabachnick BG, Fidell LS. Using multivariate statistics. 7th ed. London: Pearson Education; 2019.

46. Tomes C, Orr RM, Pope R. The impact of body armor on physical performance of law enforcement personnel: a systematic review. Ann Occup Environ Med. 2017;29(1):14.

47. Varvarigou V, Farioli A, Korre M, Sato S, Dahabreh IJ, Kales SN. Law enforcement duties and sudden cardiac death among police officers in United States: case distribution study. BMJ. 2014;349: g6534.

48. Wilmore JH, Knuttgen HG. Aerobic exercise and endurance. Phys Sportsmed. 2003;31(5):45-51. 